\title{
Manejo del dolor agudo posoperatorio en pacientes adultos no ARM
}

\section{Management of postoperative acute pain in adult non- ARM patients}

María S. Raña', Adriana Turiaci², Claudia Waisman², Débora Pellegrini ${ }^{3}$, Adriana Falak $^{4}$, Mariano de Muria ${ }^{3}$, Pablo Young $^{3}$

Fronteras en Medicina 20 I8; 13(3): I49-153. Doi: I0.31954/RFEM/20 I803/0 I49-0I53

\section{INTRODUCCIÓN}

El control satisfactorio del dolor postoperatorio es uno de los retos más importantes que permanecen sin resolver en el ámbito hospitalario, lo que motiva un fuerte impacto en los pacientes y en el sistema sanitario en su conjunto ${ }^{1}$. La mayoría de los pacientes que se someten a una intervención quirúrgica padecen dolor de diferentes características, intensidad y cortejo emocional asociado. En muchas ocasiones, se encuentra subtratado por falta de entrenamiento médico y miedos infundados de generar adicciones ${ }^{2}$. En otras ocasiones, tanto el paciente como los médicos intervinientes subestiman la importancia de este síntoma, dado que se supone una variable fisiológica luego de una intervención quirúrgica.

El dolor se define como una experiencia sensorial y emocional desagradable, asociada a un daño tisular existente o potencial. Al ser el dolor una experiencia subjetiva, para su cuantificación se requiere necesariamente de la información suministrada por el paciente, ya que los signos físicos y metabólicos son absolutamente inespecíficos y no mantienen necesariamente una relación proporcional a la magnitud del do$\operatorname{lor}^{3}$. La evaluación clínica y el empleo de escalas validadas, constituye el único método efectivo y razonable al

\footnotetext{
I. Servicio de Emergencias.

2. Servicio de Farmacia.

3. Servicio de Clínica Médica.

4.Auditoría Médica.
}

Correspondencia: Dra. María Soledad Raña. Servicio de Emergencias, Hospital Británico de Buenos Aires. Perdriel 74; CI280AEB CABA, Rep.Argentina. Email:Mariasoledad_ra@hotmail.com

Los autores declaran no poseer conflictos de intereses. pie de cama del paciente para valorar el grado de dolor posotperatorio ${ }^{3,4}$.

La implementación creciente de la evaluación estandarizada del dolor y protocolos de tratamiento y analgesia multimodal son necesarios para mejorar la calidad de atención y la satisfacción del paciente ${ }^{4,5}$. El concepto de analgesia multimodal (llamada también analgesia balanceada) se introdujo con el objetivo de mejorar la eficacia y seguridad del tratamiento, combinando fármacos y técnicas analgésicas con efectos aditivos o sinérgicos, lo que permite ahorrar opioides y disminuir la incidencia de efectos adversos medicamentosos ${ }^{6-8}$.

\section{DESARROLLO}

Dada la problemática planteada, el Comité de Farmacia y Terapéutica de nuestro hospital decidió analizar la literatura existente de relevancia y generar consenso acerca de las alternativas de manejo farmacológico del dolor agudo posquirúrgico en adultos no ventilados, mediante la generación de una guía base de recomendaciones, adaptado a la disponibilidad de fármacos de nuestro vademecum, y aplicable según el criterio del médico tratante (Tablas 1 a 4$)^{8-37}$. Dichas recomendaciones excluyen la utilización de drogas por vía peridural o adyuvantes que requieran monitorización reservada a las unidades cerradas. También se excluyeron recomendaciones de analgesia preventiva dado que dichas intervenciones se limitan al ámbito del quirófano y la evaluación prequirúrgica. Esta guía fue consensuada con los servicios clínicos y quirúrgicos, adecuándola e implementándola posteriormente.

El enfoque inicial para el tratamiento del dolor posoperatorio se basa en la estratificación del impacto doloroso según el tipo de cirugía que va a realizarse, ubicando al paciente en una de las tres columnas propuestas: bajo, moderado o alto impacto doloroso (Tabla 1). Durante el tratamiento de mantenimiento, el médico intervi- 


\begin{tabular}{|c|c|c|c|}
\hline & $\begin{array}{l}\text { QX BAIO IMPACTO DOLOROSO } \\
\text { Superficiales } \\
\text { Laparoscopias abdomen inferior } \\
\text { Cabeza y cuello, Neurocirugia } \\
\text { Pene y testículos, parto }\end{array}$ & $\begin{array}{c}\text { QX MODERADO IMPACTO } \\
\text { Laparoscopía abdomen superior } \\
\text { Laparotomía abdomen inferior } \\
\text { Discectomias mini invasivas } \\
\text { Cesáreas y ginecología } \\
\text { Próstata y drenajes torácicos } \\
\end{array}$ & $\begin{array}{c}\text { QX ALTO IMPACTO } \\
\text { Laparotomía abdomen superior } \\
\text { Lumbotomía } \\
\text { Tórax Y VATS } \\
\text { CCV } \\
\text { Ortopedia }\end{array}$ \\
\hline $\begin{array}{l}\text { DOSIS CARGA } \\
\text { (QUIROFANO) }\end{array}$ & $\begin{array}{l}\text { AINE: EV LENTO } \\
\text { DICLOFENAC } \\
\text { KETOROLAC }\end{array}$ & $\begin{array}{c}\text { BAJO IMPACTO DOLOROSO } \\
+ \\
\text { OPIOIDE DÉBIL: TRAMADOL }\end{array}$ & $\begin{array}{c}\text { BAJO IMPACTO DOLOROSO } \\
+ \\
\text { OPIOIDES FUERTES: FENTANILO } \\
\text { O MORFINA }\end{array}$ \\
\hline $\begin{array}{l}\text { DOSIS } \\
\text { MANTENIMIENTO }\end{array}$ & $\begin{array}{l}\text { DICLOFENAC } \\
\text { KETOROLAC }\end{array}$ & $\begin{array}{c}\text { BAJO IMPACTO DOLOROSO } \\
+ \\
\text { DILUCION CON TRAMADOL }\end{array}$ & $\begin{array}{c}\text { BAJO IMPACTO DOLOROSO } \\
+ \\
\text { DILUCIÓN CON FENTANILOO O } \\
\text { DILUCIÓN CON MORFINA }\end{array}$ \\
\hline
\end{tabular}

Tabla I. Enfoque inicial (se excluyen pacientes con analgesia peridural)

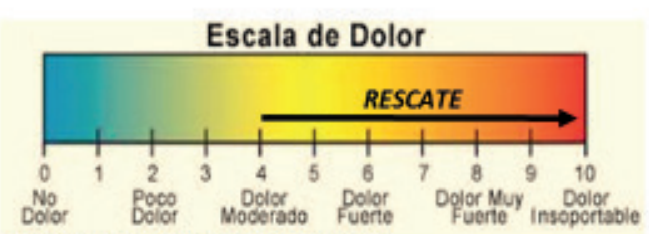

"evaluar dolor con cada toma de signos vitales

"EN PACIENTES CON DOLOR REALIZAR REEVALUACION HORARIA, haSTA EL alivio Y LUEGo espaciar RESCATES SI INTENSIDAD > 4

\section{REEVALUAR}

MANTENIMIENTO

\section{EVALUAR DOLOR}

\begin{tabular}{|c|c|c|c|}
\hline 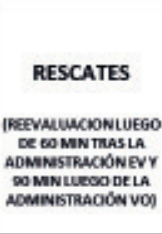 & $\begin{array}{l}\text { TRAMADOL } 50 \text { MG (DILUIDO } \\
\text { EN } 100 \text { ML SFA PASAR EN } 10 \\
\text { MIN) } \\
\text { QX MENORO PARTOS: VO }\end{array}$ & $\begin{array}{c}\text { FENTANILO SO MCG (1 AMPOLLA } \\
\text { DE S ML + SML DE SF } \rightarrow \\
\text { ADMINISTRAR } 2 \text { ML DE DICHA } \\
\text { SOLUCIÓN) } \\
0 \\
\text { MORFINA } 3 \text { MG (1 AMP + } 9 \text { ML SF } \\
\rightarrow \text { ADMINSTRAR } 3 \mathrm{ML})\end{array}$ & $\begin{array}{l}\text { FENTANILO SO MCG (1 AMPOLLA } \\
\text { DE S ML }+5 M L \text { DE SF } \rightarrow \\
\text { ADMINISTRAR } 2 \text { ML DE DICHA } \\
\text { SOLUCIÓN) } \\
0 \\
\text { MORFINA } 3 \text { MG (1 AMP }+9 \text { ML SF } \\
\rightarrow \text { ADMINSTRAR } 3 \text { ML) } \\
\text { REEVALUAR MANTENIMIENTO }\end{array}$ \\
\hline & \multicolumn{3}{|c|}{ 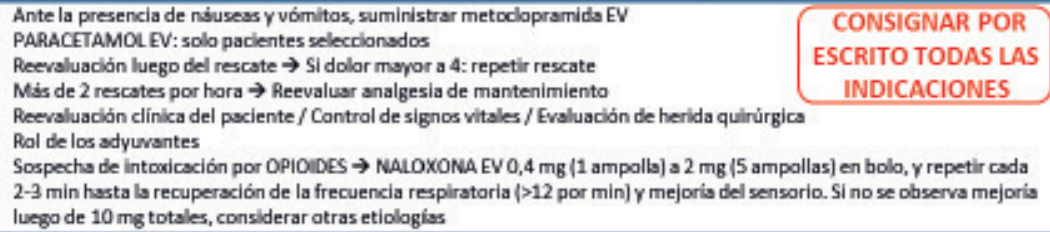 } \\
\hline
\end{tabular}

Tabla 2. Tratamiento del dolor durante la internación

\begin{tabular}{|c|c|c|c|}
\hline $\begin{array}{l}\text { MANTENIMIENTO } \\
\text { Y EGRESO }\end{array}$ & $\begin{array}{c}\text { DICLOFENAC } 75 \text { MG CADA } 12 \\
\text { HORAS } \\
0 \\
\text { IBUPROFENO } 400-600 \text { MG CADA } \\
8 \text { HORAS } \\
0 \\
\text { KETOROLAC 20 MG CADA } 8 \\
\text { HORAS (HASTA } 3 \text { DÍAS) }\end{array}$ & $\begin{array}{c}\text { DICLOFENAC } 75 \text { MG CADA } 12 \\
\text { HORAS } \\
+ \\
\text { TRAMADOL } 50 \text { MG CADA 6-8 } \\
\text { HORAS }\end{array}$ & $\begin{array}{c}\text { OPCION A: MODERADO IMPACTO } \\
\text { DOLOROSO + PARACETAMOL } 500 \\
\text { MG CADA } 6 \text { HS } \\
\text { OPCION B: CODEÍNA } 30 \text { MG + } \\
\text { PARACETAMOL } 500 \text { MG } \\
\text { (DOLOFRIX) CADA } 8 \text { HORAS + } \\
\text { DICLOFENAC } 75 \text { MG CADA } 12 \text { HS }\end{array}$ \\
\hline & $\begin{array}{l}\text { Pre acordar duración del tratamien } \\
\text { Recordar suspensión de analgésico } \\
\text { Eventual control de función renal d }\end{array}$ & $\begin{array}{l}\text { sis máxima diaria en domicilio } \\
\text { itas de seguimiento } \\
\text { ngarse el tratamiento con AINES }\end{array}$ & \\
\hline
\end{tabular}

Tabla 3. Indicación de analgesia VO de mantenimiento y al alta.

niente deberá reevaluar periódicamente al paciente, pudiendo re estratificar según la evolución clínica, la aparición de efectos adversos medicamentosos y el grado de alivio sintomático presentado. En este momento del seguimiento, toman especial relevancia la utilización de las escalas validadas para la evaluación del dolor ${ }^{38-43}$. En pacientes con valores mayores o iguales a 4 en la Escala de Valoración Numérica (EVN), está indicada la admi- 


\begin{tabular}{|l|l|l|l|l|l|l|l|}
\hline AINE & Droga & Dosis $(\mathrm{mg})$ & Intervalo $(\mathrm{hs})$ & Vía & Dosis máxima $(\mathrm{mg})$ & Comprimidos & Ampollas \\
\hline Ibuprofeno & $400-600$ & $6-8$ & VO & 2400 & $400-600$ & No disponible \\
\hline \multirow{2}{*}{ Ketorolac } & $10-30$ & 8 & $\begin{array}{l}\text { VO } \\
\text { SL } \\
\text { EV }\end{array}$ & $\begin{array}{l}\text { 90 Restricción de 72 hs } \\
(\text { VO-SL) y 48 hs (EV) }\end{array}$ & $\begin{array}{l}10-20 \\
10 \\
30\end{array}$ & 30 \\
\hline & Diclofenac & $50-75$ & 12 & VO EV & I50 & $50-75$ & 75 \\
\hline
\end{tabular}

- Alergis e hipersensibilidad.

- AINE $\rightarrow$ contraindicado en insuficiencia renal, coagulopatía, hemorragia digestiva, popccy.

- No administrar AINE dado que aumentan riesgo de efectos adversos.

- Ningún AINE es superior a otro $\rightarrow$ elegir según el paciente (edad/comorbilidades)

- Ketorolac: mayor riesgo de insuficiencia renal y hemorragia digestiva (precaución en ancianos).

- Sinergismo con paracetamol.

- Aumento del riesgo de SCA y ACV en pacientes con factores de riesgo cardiovascular (FDA 20I5).

\section{PARACETAMOL}

- Efecto analgésico y antipirético, sin efecto antiinflamatorio.

- Inicio de acción: 5 minutos (EV) - 35 minutos (VO).

- Duración del efecto analgésico: 4-6 horas.

- Dosificación: Dosis máxima en adultos: 4 g/día. Pacientes de alto riesgo para hepatopatías: 3 g/día. Administrar la dosis diaria dividida en intervalos entre dosis de 6-8 horas.

- Contraindicaciones: hipersensibilidad al principio activo e insuficiencia hepática avanzada.

- Puede utilizarse en embarazo y lactancia.

Presentaciones disponibles:

Endovenoso (Paracetamol KABI $10 \mathrm{mg} / \mathrm{ml}$ ): frascos ampolla de $1000 \mathrm{mg}$ ( $100 \mathrm{ml}$ ) y $500 \mathrm{mg}$ (50 ml); se infunde en 15 minutos (sin diluir).

Oral: comprimidos de 500-1000 mg.

\section{$\triangle$ NO SOBREPASAR LA DOSIS DIARIA}

\section{OPIOIDES}

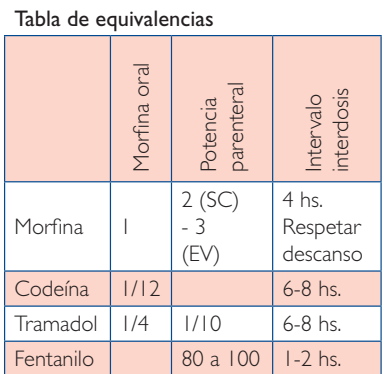

\begin{tabular}{|c|c|c|c|c|c|c|}
\hline & Opioide & $\begin{array}{l}\text { Vida media } \\
\text { (inicio de acción) }\end{array}$ & Presentación & Dosis & Comentarios & $\begin{array}{l}\text { Efectos adversos } \\
\text { comunes }\end{array}$ \\
\hline \multirow[t]{2}{*}{ Fuertes } & Morfina & $2-4$ hs. (5 min) & $\begin{array}{l}\text { Ampolla de } 10 \mathrm{mg} / \\
\mathrm{ml} ; \text { jarabe al } 1 \%(10 \\
\mathrm{mg} / \mathrm{ml}) \text { y } 2.5 \%(25 \\
\mathrm{mg} / \mathrm{ml})\end{array}$ & $\begin{array}{l}\text { Bolo y rescate: I-3 } \\
\text { mg cada } 5 \text { minutos } \\
\text { hasta el alivio del do- } \\
\text { lor o aparición de } \\
\text { RAM. Infusión: I-5 } \\
\text { mg/h (diluido en dx } \\
5 \% \text { o SF). }\end{array}$ & Reducir dosis en $\mathbb{R}$ & \multirow{4}{*}{$\begin{array}{l}\text { Náuseas, vómi- } \\
\text { tos, neurotoxicidad, } \\
\text { somnolencia, re- } \\
\text { tención aguda de } \\
\text { orina, constipación } \\
\text { (no genera tole- } \\
\text { rancia). }\end{array}$} \\
\hline & Fentanilo & $2-4$ hs (3-4 min) & $\begin{array}{l}\text { Ampolla de } 350 \\
\mathrm{mcg} / 5 \mathrm{ml}\end{array}$ & $\begin{array}{l}\text { Bolo y rescate: } 50- \\
100 \text { mcg hasta alivio } \\
\text { del dolor o aparición } \\
\text { de RAM. Infusión: } \\
100 \text { mcg/h (diluido } \\
\text { en dx 5\% o SF) }\end{array}$ & $\begin{array}{l}\text { Mayor potencia y } \\
\text { menor liberación de } \\
\text { histamina que mor- } \\
\text { fina.Vida media se } \\
\text { prolonga con la infu- } \\
\text { sión continua }\end{array}$ & \\
\hline \multirow[t]{2}{*}{ Débiles } & Tramadol & $\begin{array}{l}6-8 \text { hs (EV } 15 \\
\text { min }-V O 60 \\
\text { min) }\end{array}$ & $\begin{array}{l}\text { Ampollas de } 50 \text { y } \\
100 \text { mg tramadol. } \\
\text { Comprimidos: } 50 \mathrm{mg} \text {. } \\
\text { Gotas: ( } 100 \mathrm{mg}) \text { :Tra- } \\
\text { ma Klosidol } 28 \text { gotas } \\
\text { Tramal } 40 \text { gotas (*) }\end{array}$ & $\begin{array}{l}\text { Dosis máxima } 400 \\
\text { mg/día (en IR y an- } \\
\text { cianos hasta } 200 \\
\text { mg día) }\end{array}$ & & \\
\hline & Codeína & $\begin{array}{l}2.5 \text { a } 5 \text { hs (30- } \\
60 \text { min) }\end{array}$ & $\begin{array}{l}\text { Comprimidos con } 50 \\
\text { mg de paracetamol } \\
\text { (Dolofrix) y } 60 \text { mg + } \\
300 \text { mg de paraceta- } \\
\text { mol (Dolofrix forte) }\end{array}$ & $\begin{array}{l}\text { Dosis máxima } 360 \\
\text { mg/día }\end{array}$ & $\begin{array}{l}\text { Se metaboliza a } \\
\text { morfina. Efecto an- } \\
\text { titusivo }\end{array}$ & \\
\hline
\end{tabular}

\section{ADYUVANTES}

\section{Pregabalina}

- Biodisponibilidad oral $<90 \%$.

- Farmacocinética lineal, lo que permite aumentar la dosis progresivamente cada 48 horas.

- Excreción renal - ajustar dosis en IR

- Dosis: 50-600 mg/día VO dividido en dos o tres tomas.

\section{Analgesia multimodal}

La combinación con opioides suaves disminuye hasta un $30 \%$ la dosis de las mismas.

La coadministración con AINE proporciona sinergismo analgésico por la sumatoria de mecanismos de acción.

\section{Indicaciones para la vía EV:}

- Dolor agudo cuando las otras vías de administración no se encuentran disponibles en pacientes con contraindicación para recibir AINE.

- Dolor agudo en pacientes con insuficiencia renal (clearance de creatinina $<60 \mathrm{ml} / \mathrm{min} / 1.73 \mathrm{~m} 2$ ).

- Antipirérico en pacientes con contraindicación para recibir AINE (oncohematológicos, insuficiencia renal).
- Indicado en dolor pop con componente neuropático y pacientes con antecedente de consumo previo.

- Efectos adversos: edemas aumento de peso, fatiga, somnolencia, mareos, cefaleas, náuseas, vómitos.

Abreviaturas: RAM: reacción adversa a medicamento. IR: insuficiencia renal. D× 5\%: dextrosa 5\%. SF: solución fisiológica. // (*) otras marcas $\rightarrow$ consultar.

Tabla 4. Algunas consideraciones sobre AINE, paracetamol, opioides y adyuvantes.

nistración de rescate analgésico y posterior control clínico. De requerir más de dos rescates en una hora, se

sugiere la reevaluación del esquema de mantenimiento

(Tabla 2). Cuando el paciente se encuentre en condi- 
ciones de egreso hospitalario, se sugieren varias opciones para el tratamiento ambulatorio, siempre con indicaciones claras y fecha de finalización preacordadas entre el médico y el paciente, para limitar los efectos adversos que puedan aparecer con el tratamiento prolongado (Tabla 3).

En la Tabla 4 se detallan algunas consideraciones farmacocinéticas y farmacodinámicas de las drogas inclui- das en las recomendaciones, así como las presentaciones disponibles en nuestra Farmacia de internación.

Entre los objetivos futuros del Comité, se encuentran la revisión y actualización periódica de la guía, la incorporación de nuevos fármacos o técnicas analgésicas, siendo de especial interés la medición de la adherencia en la implementación de la guía en la práctica cotidiana y el estímulo de su utilización.

\section{BIBLIOGRAFÍA}

I. Gallego JI, Rodríguez de la Torre MR, Vázquez-Guerrero JC, Gil M. Estimation of the prevalence and severity of postoperative pain and relation with patient satisfaction. Rev Soc Esp Dolor 2004; I 1:197-202

2. Kalkman CJ, Visser K, Moen J, Bonsel GJ, Grobbee DE, Moons KGM. Preoperative prediction of severe postoperative pain. Pain 2003; 105:415-23.

3. American Pain Society Reviews. Pain:The fifth vital sign (2002). http:// www.ampainsoc.org/advocacy/fifth (consultado el I5/03/2018)

4. Hutchinson RW. Challenges in acute post-operative pain management. Am J Health-Syst Pharm 2007; Vol 64(I5):Suppl 4.

5. White PF, Kehlet H. Improving Postoperative Pain Management. Anesthesiology 20 I0; 12:220-5

6. Kehlet H, Dahl JB.The value of "multimodal" or "balanced analgesia" in post-operative pain treatment. Anesth Analg 1993;77: I048-56.

7. Helander EM, Menard BL, Harmon C, et al. Multimodal Analgesia, current concepts, and acute pain considerations. Curr Pain Headache Rep 2017;21 ( I):3.

8. Elvir-Lazo OL, White PF.The role of multimodal analgesia in pain management after ambulatory surgery. Curr Opin Anaesthesiol 2010;23:697-703.

9. González de Mejía N. Postoperative multimodal analgesia. Rev Soc Esp Dolor 2005; 12 : 11 2- 18

10. Postoperative Pain Management.NHS Quality Improvement Scotland 2004. www.nhshealthquality.org (consultado el 03/03/20 I 8).

I I. Lovich-Sapola J, Smith CE, Brandt CP. Postoperative Pain Control. Surg Clin North Am 2015;95(2):301- I 8.

12. Jacobson JG. Pain management in ambulatory surgery-A Review. Pharmaceuticals 2014;7:850-65.

13. American Society of Anesthesiologists Task Force on Acute Pain Management. Practice guidelines for acute pain management in the perioperative setting: an updated report by the American Society of Anesthesiologists Task Force on Acute Pain Management. Anesthesiology 2012; | 16:248-73.

14. Graham GG, Davies MJ, Day RO, et al. The modern pharmacology of paracetamol: Therapeutic actions, mechanism of action, metabolism, toxicity and recent pharmacological findings. Inflammopharmacology 2013;21:201-32

15. Barden J, Edwards J, Moore A, et al. Single dose oral paracetamol (acetaminophen) for postoperative pain. Cochrane Database Syst Rev 2004; I:CD004602.

16. Apfel CC, Souza K, Portillo J, et al. Patient satisfaction with intravenous Acetaminophen: A Pooled Analysis of Five Randomized, Placebo-Controlled Studies in the Acute Postoperative Setting. I Healthc Qual 2015;37(3): I55-62

17. Gandhi K, Baratta JL, Heitz JW, et al. Acute pain management in the postanesthesia care unit.Anesthesiology Clin 20 I 2;30:e I -e I 5.

18. Bekawi MS, El Wakeel LM, Al Taher WM, Mageed WM. Clinical study evaluating Pregabalin efficacy and tolerability for pain management in patients undergoing laparoscopic cholecystectomy. Clin J Pain 2014; 30( I I):944-52.

19. Sarakatsianou C, Theodorou E, Georgopoulou S, et al. Effect of pre-emptive pregabalin on pain intensity and postoperative mor- phine consumption after laparoscopic cholecystectomy. Surg Endosc 20 | 3:27:2504- I I.

20. Yu L, Ran B, Li M, et al. Gabapentin and pregabalin in the management of postoperative pain after lumbar spinal surgery: A systematic review and meta-analysis. Spine 2013;38:1947-52.

21. Sen $H$, Sizlan A, Yanarates $O$, et al.The effects of gabapentin on acute and chronic pain after inguinal herniorrhaphy. Eur J Anaesthesiol 2009;26:772-76.

22. Dauri M, Faria S, Gatti A, et al. Gabapentin and pregabalin for the acute post-operative pain management. A systematic-narrative review of the recent clinical evidences. Curr Drug Targets 2009; 10:716-33.

23. Misiołek H, Cettler M, Woron M, et al. The 2014 guidelines for post-operative pain management. Anaesthesiol Intensive Ther 2014; 46 (4):221-44.

24. Oderda G. Challenges in the management of acute postsurgical pain. Pharmacotherapy 2012; 32(9):6S-IIS

25. Allegri M, Grossi P. Management of postoperative pain: how accurate and successful is our acute pain management? Minerva Anestesiol 2012;78:1.

26. Mathiesen $\mathrm{O}$, Thomsen BA, Kitter B, et al. Need for improved treatment of postoperative pain. Dan Med J 2012;59:A440I.

27. Duncan F, Day R, Haigh C, et al. First steps toward understanding the variability in acute pain service provision and the quality of pain relief in everyday practice across the united kingdom. Pain Medicine 20|4; 15: | 42-53.

28. Grond S, Sablotzki A. Clinical pharmacology of tramadol. Clin Pharmacokinet 2004; 43:879-923.

29. Pommergaard HC, Klein M, Burcharth J, et al. Variation in postoperative non-steroidal anti-inflammatory analgesic use after colorectal surgery: a database analysis. BMCAnesthesiology 20 |4; | 4:8.

30. Pardo C, Muñoz C, Chamorro C y grupo de trabajo de analgesia y sedación de la SEMICYUC. Monitorización del dolor. Recomendaciones del grupo de trabajo de analgesia y sedación de la SEMICYUC. Med Intensiva 2006;30(8):379-85.

31. Esteve Pérez N, Usoles EdR, Giménez Jiménez R, et al. Seguridad y efectividad del tratamiento del dolor agudo postoperatorio: seguimiento de 3.670 pacientes. Rev Esp Anestesiol Reanim 2008:55:54|-47.

32. White PF, Kehlet $\mathrm{H}$. Improving postoperative pain management. Anesthesiology 2010; I 12:220-5.

33. Kuusniemmi K, Poyhia R. Present-day challenges and future solutions in postoperative pain management: results from PainForum 2014. Journal of Pain Research 2016;(9):25-36.

34. Katz J,Weinrib A, Fashler SR, et al.The Toronto General Hospital Transitional Pain Service: development and implementation of a multidisciplinary program to prevent chronic postsurgical pain. Journal of Pain Research 2015;(8):695-702.

35. Schug SA, Monopas A. Update on the role of non-opioids for postoperative pain treatment. Best Pract Res Clin Anaesthesiol 2007;(21): I 5-30.

36. Hui Yun V, Abrishami A, Peng PHP, et al. Predictors of postoperative pain and analgesic consumption. Anesthesiology 2009; ( I I ):657-77. 
37. Carroll I, Hah J, Mackey S, et al. Perioperative interventions to reduce chronic postsurgical pain. J Reconstr Microsurg. 2013;29(4):213-22.

38. Stasiowska MK,Su Cheen NG, GubbayAN, etal.Postoperative pain management. Brit Jour of Hospital Medicine 20 I 5;76( I 0):570-5

39. Chapman CR, Casey KL, Dubner R, et al. Pain measurement: an overview. Pain 1985;22: I-31.

40. Ho K, Spence J, Murphy MF. Review of pain-measurement tools. Ann Emerg Med. 1996;27:427-32
41. Ahlers SJ, van Gulik L, van derVeen AM, et al. Comparison of different pain scoring systems in critically ill patients in a general ICU. Crit Care 2008; I2:RI5

42. Li Li MSN, Xueqin Liu BSN, Herr K. Postoperative pain intensity assessment: A comparison of four scales in chinese adults. Pain Med 2007;8(3):223-34

43. Hawker GA, Mian S, Kendzerska T, et al. Measures of adult pain. Arthritis Care \& Research 20 I I;63( I I):240-525. 